\title{
DISTRIBUIÇÃO GEOGRÁFICA E CARACTERIZAÇÃO DA PRODUÇÃO DE RÃ-TOURO Lithobates catesbeianus NO ESTADO DE RONDÔNIA (BRASIL)
}

Geographical distribution and characterization of the Bullfrog production Lithobates catesbeianus in the state of Rondônia (Brazil)

Distribución geográfica y caracterización de la producción de Rana Toro Lithobates catesbeianus en el estado de Rondônia (Brasil)

\author{
Rafael Camoleze Maltarolo ${ }^{1}$, Raniere Garcez Costa Sousa ${ }^{* 1}$ \\ ${ }^{1}$ Departamento de Engenharia de Pesca, Programa de Pós-Graduação em Ciências Ambientais, Universidade \\ Federal de Rondônia, Presidente Médici, Rondônia, Brasil.
}

*Correspondência: Universidade Federal de Rondônia (UNIR) - Programa de Pós-Graduação em Ciências Ambientais (PGCA), Rua da Paz, 4376 - Bairro Lino Alves Teixeira - CEP: 76.916-000, Caixa Postal 32, Presidente Médici-Rondônia,Brasil.email ranieregarcez@unir.br

Artigo recebido em 07/11/2017 aprovado em 18/02/2019 publicado em 30/03/2019.

\section{RESUMO}

A atividade ranícola tem afirmado o Brasil como o segundo maior produtor mundial de rã. Contudo, existem poucos dados estatísticos oficiais que abordam sobre esta atividade no país. E, tratando-se da região Norte do Brasil, especialmente no estado de Rondônia, pouco ou nenhum estudo foi encontrado abordando essa temática. A presente pesquisa realizou um diagnóstico dos ranários no estado de Rondônia, visando registrar informações desse seguimento primário de produção. Os dados foram coletados utilizando o método interrogativo, com o uso de questionários semiestruturados junto aos proprietários de ranários. Foram localizadas duas raniculturas no estado, que atuam com fins comerciais, no sistema anfigranja de criação, estas possuem capacidade produtiva em torno de 5 ton. ano $^{-1}$, com uma venda média semanal de $50 \mathrm{~kg}$ de carne de rãs. Os preços dos produtos variam conforme o tipo: carcaça inteira e congelado $R \$ 60,00$, inteiros frescos $R \$ 50,00$ ou corte específico no mercado varejista. A criação de rã no Estado de Rondônia é de pequeno porte, e sua produção é comercializada em lanchonetes e restaurantes nos municípios de origem e cidades vizinhas como Cacoal e Ariquemes.

Palavras-chave: Comercialização. Ranicultura. Sistema de criação.

\section{ABSTRACT}

Frog farming activity has affirmed the Brazil as the second largest producer of frog in the world. However, there is little official statistical data on this activity in the country. In addition, in the Northern region of Brazil, especially in the State of Rondonia, little or no study was found addressing this theme. This research carried out a diagnosis of the Frog farming in the state of Rondônia, aiming to record information on this primary production sector. Data were collected using the interrogative method, with the use of semi-structured questionnaires with the ranch owners. Two ranches (Frog farming) were located in the state, which operate with commercial purposes, in the "Amphifarm" system production, these farms have a productive capacity around 5 tons. year ${ }^{-1}$, with a commercialization rounding $50 \mathrm{~kg}$ of frogs meat by weekly. The prices of products vary according to type: whole and frozen animal $R \$ 60.00$, fresh whole $R \$ 50.00$ or specific cut in the trade in the market. Frog farming in the State of Rondônia acts in smallscale production, and its production is marketed in snack bars and restaurants in the municipalities of origin and neighboring cities such as Cacoal and Ariquemes.

Keywords: Commercialization. Ranch-Frog farming. Creation system.

\section{RESUMEN}

La actividad productora del rana ha afirmado a Brasil como el segundo mayor productor mundial del ranas. Sin embargo, existen pocos datos estadísticos oficiales que abordan sobre esta actividad en el país. $Y$, tratándose de la región Norte de Brasil, especialmente en el estado de Rondônia, poco o ningún estudio fue encontrado abordando esa temática. La presente investigación realizó un diagnóstico de los ranarios en el estado de Rondônia, buscando registrar informaciones de ese seguimiento primario de producción. Los datos fueron recolectados utilizando el 
método interrogativo, con el uso de cuestionarios semiestructurados junto a los propietarios de las haciendas. Se localizaron dos granjas ranícolas en el estado, que actúan con fines comerciales, en el sistema anfigranja de cría, éstas poseen capacidad productiva en torno a 5 ton. año ${ }^{-1}$, con una venta media semanal de $50 \mathrm{~kg}$ de carne de ranas. Los precios de los productos varían según el tipo: animal entero y congelado $R \$ 60,00$, enteros frescos $R \$ 50,00$ o corte específico en el mercado consumidor. La creación de rana en el Estado de Rondônia es de pequeño porte, y su producción se comercializa en cafeterías y restaurantes en los municipios de origen y en ciudades vecinas como Cacoal y Ariquemes.

Descriptores: Comercialización. Ranario. Sistema de creación.

\section{INTRODUÇÃO}

No Brasil, a criação de rãs teve início em 1935, quando o então técnico canadense Tom Cyrill Harrison instalou o primeiro ranário no estado do Rio de janeiro, importando 300 animais da espécie Rana catesbeiana, Shaw (1802) dos Estados Unidos da América (Ranário Aurora, 1938; VIZZOTO, 1979).

Na década de 1970, o hábito de consumir rã não contemplava as famílias de baixa renda, estas ficando restritas apenas aos animais capturados na natureza, de onde tiravam proteína animal para o complemento da alimentação familiar (CARRARO, 2008). Com a valorização da carne de rã no mercado nacional na década de 80 , muitos empreendedores rurais foram atraídos a investirem na criação desses animais (FEIX et al., 2006; RODRIGUES et al., 2010).

Dentre as espécies de anfíbios, Leptodactylus latrans, Linnaeus (1758) (rã-manteiga) (FIGUEIREDO et al., 2001), Leptodactylus labyrinthicus, Spix (1824) (rã-pimenta) (AGOSTINHO et al., 1991) e Lithobates catesbeianus, Shaw (1802) (rã-touro) (MANSANO et al., 2012), com potencialidade zootécnica, domesticação e rentabilidade ao produtor no início da atividade ranícola no Brasil, a L. catesbeianus, estabeleceu-se como a principal espécie utilizada nos ranários comerciais brasileiros (MOREIRA, 2011), em virtude de sua fácil adaptação a sazonalidade climática do país, e por suas caracteristicas zootécnicas ideais para o cultivo, tais como: precocidade, fecundidade e prolificidade (CARRARO, 2008; ANTONUCCI, 2009).

No entanto, o cultivo dessa espécie ocorria no país, de forma inadequada, sobretudo em instalações improprias com mão de obra não especializada (FEIX et al., 2006; DIAS; RODRIGUES et al., 2010), fatores que contribuíram com o declínio da produção nesse setor (LIMA E AGOSTINHO, 1992) especialmente com o fechamento de 1600 raniculturas nos anos 80 (LIMA e AGOSTINHO, 1989) restando apenas 600 empreendimentos na década seguinte (LIMA et al., 1999). Por outro lado, a redução no número de raniculturas na década de 80 , não foi suficiente para impedir o crescimento do setor, que aumentou sua produção em torno de $2600 \%$ no período de 1989 a 2001 (FAO, 2010), constituindo, assim, um indicativo de recuperação e crescimento das raniculturas no país (DE OLIVEIRA, 2015).

A ranicultura brasileira se manteve nos últimos dez anos, com uma produção média de carne de rã de 600 ton. ano ${ }^{-1}$ (IBAMA, 2009, FAO, 2010), procedente principalmente dos estados de São Paulo, Minas Gerais e Rio de Janeiro, responsáveis por 69\% da produção, seguidos dos estados de Goiás e Distrito Federal com 24,7\%, Santa Catarina com 4,2\%, Bahia e Ceará com 1,3\% da produção total nacional. Dessa forma, a somatória da produção de rã desses estados, tem assegurado ao país o ranking de segundo maior produtor mundial de carne de rã (DE OLIVEIRA, 2015). 
A região Norte do Brasil, especialmente o estado de Rondônia, apresenta ótimas condições climáticas e sazonais para o desenvolvimento e exploração do cultivo de rãs (LIMA e AGOSTINHO, 1992), no entanto, não existem dados oficiais sobre a produção dessa matéria-prima, nem informações técnicas relacionadas aos ranários do estado.

Dessa forma, este estudo realizou um diagnóstico dos ranários no estado de Rondônia, visando identificar e registrar informações técnicas, relevantes desse seguimento primário de produção, com a finalidade de subsidiar informações do atual perfil socioeconômico desse setor produtivo para as comunidades técnica, científica e atuantes da produção de rãs.

\section{MATERIAIS E MÉTODOS}

\section{Área de estudo}

O estado de Rondônia possui 52 municípios e ocupa uma área de 237.590,547 km² (IBGE, 2017), apresenta condições térmicas anuais que oscilam entre 18 a $33^{\circ} \mathrm{C}$ (INMET, 2017). Nessa região, foram localizadas 02 (duas) raniculturas em atividade no estado de Rondônia. Uma localizada no município de

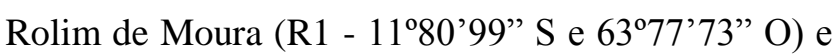
outra no município de Monte Negro (R2 - 104’27” S e $63^{\circ} 20^{\prime} 2^{\prime \prime} \mathrm{O}$ ) figura 1.

Figura 1. Localização das raniculturas nos municípios de Rolim de Moura (R1) e Monte Negro (R2) no estado de Rondônia. A rodovia de acesso as raniculturas está destacada em vermelho no mapa.

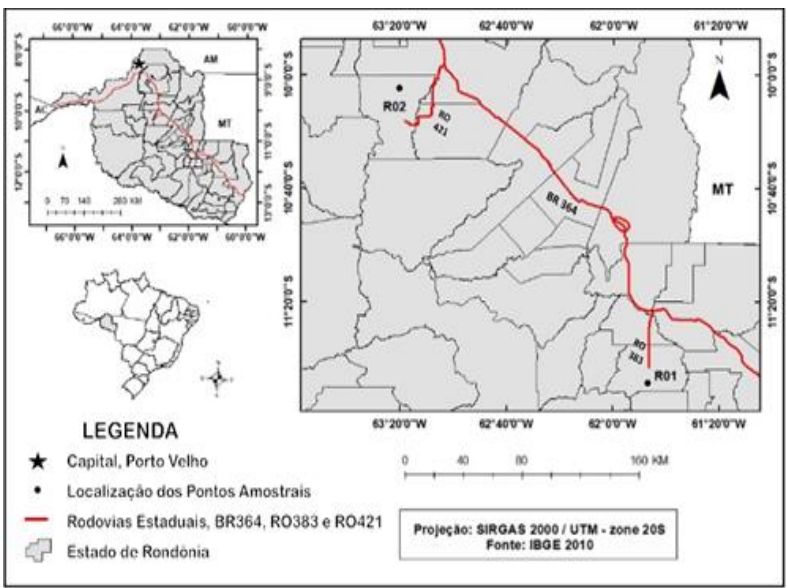

\section{Procedimento amostral e coleta dos dados}

O diagnóstico sobre o perfil socioeconômico das raniculturas do estado de Rondônia, ocorreram em duas etapas: I) realização do levantamento dos ranários existentes na região do estudo, através de informações oriundas de instituições públicas e reguladoras: Agência de Defesa Sanitária Agrosilvopastoril do Estado de Rondônia - IDARON, Empresa de Assistência Técnica e Extensão Rural do Governo de Rondônia - EMATER-RO, Secretaria de Estado do Desenvolvimento Ambiental - SEDAM e Instituto Brasileiro do Meio Ambiente e dos Recursos Naturais Renováveis - IBAMA; e II) visita em campo, para as aplicações de entrevistas junto aos ranicultores.

O método utilizado para a coleta de dados foi o interrogativo (BARDIN, 1977; OLIVEIRA, 2008; KAUFMANN, 2013). Foram elaborados formulários semiestruturados com 30 perguntas e aplicados juntos aos produtores de rãs, no intuito de confrontar as informações entre si, os quesitos abordaram temas relacionados ao perfil socioeconômico dos ranicultores: renda da família, diversificação da propriedade, experiência no ramo de criação, mão de obra do empreendimento; sua distribuição espacial; caracterização do modelo de criação: custo de produção, insumos utilizados, capitação de água, capacidade de lotação atual do empreendimento, problemas sanitário, área de ampliação, aquisição dos animais e manejo; e o destino da comercialização da produção de carne de rã no estado: nichos e expansão de mercado, abate, licenciamento da propriedade, mercado consumidor, no período de março a abril de 2017.

\section{Análises estatística dos dados}

As análises estatísticas foram de cunho descritivo (REIS et al., 2002), utilizada essencialmente para uma melhor visualização dos dados (médias e 
desvio padrão) diante do estado atual da ranicultura na região do estudo.

\section{RESULTADOS}

\section{Perfil socioeconômico da produção de rã}

As pesquisas junto aos órgãos públicos $\mathrm{e}$ agências de apoio técnico rural, permitiram o acesso as informações relacionadas a localização de duas raniculturas, estas, confirmadas durante as visitas em campo, estando localizadas nos municípios de Rolim de Moura (R1) e Monte Negro (R2).

Nessas raniculturas, os recursos empregados para a produção dos animais são próprios, e utilizam a mão de obra familiar e empregatícia nas atividades de manejo e comercialização, sendo que a criação de rã não é considerada a principal atividade da propriedade.

As estruturas das instalações das raniculturas (R1 e R2) são construídas de alvenaria, e possuem áreas de $225 \mathrm{~m}^{2}$ e $128 \mathrm{~m}^{2}$, respectivamente, destinadas exclusivamente para as atividades de produção.

O sistema de criação empregado nessas propriedades é o de Anfigranja, compondo em suas instalações repartições para os setores de reprodução, girinagem e engorda, este último, fecha um ciclo de produção com 7 meses para R1 e com 10 meses para $\mathrm{R} 2$, incidindo em peso final para o abate dos animais com uma média de $280 \mathrm{~g}$ e $450 \mathrm{~g}$, respectivamente.

A capacidade de cultivo das raniculturas $\mathrm{R} 1 \mathrm{e}$ R2, foi estimada em 12.000 e 8.400 rãs, com densidade média dos animais de 88 rãs $/ \mathrm{m}^{2}$ e 100 rãs $/ \mathrm{m}^{2}$, da fase de engorda até o abate, correspondendo a uma produção média de 2.600 e 2.400 kg.ano ${ }^{-1}$, respectivamente.

Os respectivos ranários, mantém estocados de 20 a 30 casais em seus setores de reprodução, respeitando a estocagem para o povoamento de machos e fêmeas, na proporção de (1:1) um macho para uma fêmea, com densidade de 1,7 rãs $/ \mathrm{m}^{2}$ e 2 rãs $/ \mathrm{m}^{2}$, simultaneamente. Dessa forma, a criação de rã é mantida durante todo o ano para atender a procura pelos produtos provenientes dessa produção.

\section{Origem dos animais, manejo e custo de produção}

Os primeiros casais de matrizes e reprodutores, e girinos adquiridos pelos proprietários dos ranários R1e R2 (100\% dos animais) para essa produção foram originários do município de Santa Isabel, estado de São Paulo adquiridos com preço de $\mathrm{R} \$ 90,00$ o casal e $\mathrm{R} \$ 650,00$ o milheiro de girinos (sementes).

A água utilizada nos ranários, para o manejo e manipulação dos animais durante o cultivo, é originaria de represas e poços dentro das propriedades, de onde são bombeadas até os sistemas de produção. $\mathrm{O}$ uso de energia elétrica nesse processo, contribui em um custo mensal em torno de $\mathrm{R} \$ 200,00$ para a ranicultura $\mathrm{R} 1$ e $\mathrm{R} \$ 75,00$ para a $\mathrm{R} 2$.

A alimentação dos planteis, são realizadas com o uso de ração comercial, com teor proteico diferenciado para cada fase de cultivo: I) fase de girinagem e imago ração de $45 \%$ de proteína bruta (PB) valor de $\mathrm{R} \$ 9,00$ por $\mathrm{kg}$, e II) fase de engorda com $40 \%$ (PB), valor de $\mathrm{R} \$ 2,75$ por kg de ração.

\section{Comercialização e escoamento da produção}

Cada empreendimento, produz em torno de 50 $\mathrm{kg}$ de carne de rãs por semana. Na ranicultura $\mathrm{R} 1$, os preços dos produtos variam conforme o tipo: se for comercializado o animal inteiro e congelado (eviscerado, com 3 a 4 peças) o quilo da carne custa $\mathrm{R} \$ 60,00$. Se a especiaria for composta somente por coxas de rãs, o preço sobe para $R \$ 90,00 / \mathrm{kg}$. Por outro lado, na ranicultura $\mathrm{R} 2$, o preço médio para a comercialização de indivíduos inteiros frescos e ou 
congelados no comércio varejista, custam em torno de $\mathrm{R} \$ 50,00 / \mathrm{kg}$, contendo de 2,5 a 3 peças por quilo.

Os ranários avaliados ainda estão em processo de certificação no SIE (Serviço de Inspeção Estadual) que autoriza a comercialização interestadual da carne de rã e do SIF (Serviço de Inspeção Federal), que permite a comercialização internacional de seus produtos.

Os principais clientes são os estabelecimentos privados, que são administrados por pequenos comerciantes, restaurantes e lanchonetes, que compram os produtos dos ranicultores de Rondônia.

Num cenário geral da distribuição da carne de rã no estado de Rondônia, pode-se dizer que, o destino principal dos produtos de R1 e R2 é (100\%) comercializado e consumido no próprio estado. Do total produzido pelos produtores, cerca de 85 e $10 \%$, são comercializados na cidade de origem (Rolim de Moura e Monte Negro), e os outros 15 e 90\%, vem sendo comercializados nos varejos das cidades vizinhas como: Cacoal (R1) e Ariquemes (R2), havendo ainda um nicho de mercado não explorado como a capital do estado Porto Velho e cidades do interior, de expressão econômica como Ji-Paraná e Vilhena.

\section{DISCUSSÃO}

Estudos relacionados a produção da carne de rã no Brasil, ainda são escassos, mas tem ocorrido lentamente nos últimos 30 anos (OLIVEIRA, 1982; FONTANELLO et al., 1988; LIMA \& AGOSTINHO, 1989, 1999; FERREIRA et al., 2002; CRIBB et al., 2013). A criação de répteis somada a ranicultura representam tão-somente $0,1 \%$ da produção aquícola no país (IBGE, 2015).

Por outro lado, a ranicultura tem se destacando, e no ano de 2015 exibiu uma produção estimada em 631 toneladas, gerando um ganho comercial equivalente a $\mathrm{R} \$ 2,26$ milhões (IBGE, 2015). Essa produção, concentrou-se principalmente na região Sudeste do país, onde o estado de São Paulo liderou o mercado nacional com $69 \%$ do total registrado (OSTRENSKY \& SOTO, 2008). No entanto, essa realidade não é a mesma para a região Norte, onde somente o estado de Rondônia contribuiu com essa estatística de produção, registrado uma média de 5 ton.ano ${ }^{-1}$ de carne de rã, representando unicamente $0,79 \%$ do total nacional (GOMES, 2008).

A região Norte do país apresenta plenas condições hidrológicas, climáticas e geográficas ideais para o desenvolvimento da criação da rã-touro (LIMA e AGOSTINHO, 1992; DE OLIVEIRA, 2015), especialmente por serem animais heterotérmicos e necessitam de ambiente úmido e com fonte de calor abundante (BRAGA e LIMA, 2001; LIMA et al., 2003; JUNIOR et al., 2005) sendo a faixa ideal para um bom desenvolvimento da rã-touro entre 25 e $28{ }^{\circ} \mathrm{C}$ (MAZZONI, 2001; MORETTO et al., 2013).

São vários os sistemas de engorda utilizados para a criação de rã (MOREIRA, 2011), no entanto, os mais utilizados são: Confinamento (OLIVEIRA, 1982), Inundado (CRIBB et al., 2013) e Anfigranja (LIMA et al., 1999; FEIX et al., 2006; CARVALHO; MOREIRA, 2011), este último é empregado na região do estudo, assim como em mais de $55 \%$ dos ranários existentes e em atividade no país (COLOMBANO, 2005). Este sistema de criação, eram tradicionais nos estados de São Paulo e Rio de Janeiro, onde consistiam basicamente de raniculturas compostas por baias em piso de cimento, uma piscina central, abrigos e comedouros (FONTANELLO et al., 1993; LIMA e AGOSTINHO, 1999; CASTRO, 2010; CARVALHO; MOREIRA, 2011).

Os entrevistados consideram a ranicultura uma atividade secundária, onde utilizam a mão-de-obra familiar como elementar para o desenvolvimento 
dessa atividade. O envolvimento de integrantes da família na criação de rãs, são frequentes também, em outras regiões do país, como reportado no Rio Grande do Sul (FEIX et al., 2006), São Paulo (CORRÊA et al., 2008; MOREIRA, 2011; AFONSO, 2012) e Rio de Janeiro (BORIN et al., 2013).

Os preços dos produtos oriundos das raniculturas ofertados no mercado varejista, variam entre as regiões do país, como observado por Cribb et al. (2009) que registraram no ano de 2003, a variação do preço da rã inteira e congelada vendida no Mercado do Produtor da Barra da Tijuca, no Rio de Janeiro, com valores entre $R \$ 34,00 / \mathrm{kg}$ a $\mathrm{R} \$ 40,00 / \mathrm{kg}$, e pode ser encontrado por valores que vão de $\mathrm{R} \$ 40,00$ a $75,00 / \mathrm{kg}$ nos grandes centros urbanos (AFONSO, 2012). Ainda, Feix et al. (2006) também reportaram para o estado do Rio Grande do Sul, valores de venda da carne de rã em torno de $\mathrm{R} \$ 30,00 / \mathrm{kg}$. Os valores de venda da carne de rãs, registrados por ambos os grupos de autores, são inferiores aos mencionados no presente estudo, e essas diferenças nos preços podem estar relacionadas aos diferentes períodos de comercialização, localização geográfica dos ranários e a aceitabilidade do produto pelo mercado consumidor (CRIBB; DE CARVALHO; MENDONÇA, 2009; AFONSO, 2012).

Os produtos dos ranicultores Rondonienses apresentam características morfometrias aptas para exportação. Embora a certificação dos produtores no SIE e no SIF tenha sido um entrave na comercialização dos produtos, pelo fato de que os dois empreendimentos, ainda comercialização seus produtos sem os selos de certificação, correndo sérios risco de serem autuados, o que tem dificultado a expansão da atividade e comercialização além das barreiras regionais (GOMES, 2008). No entanto, essa situação é muito comum entre os criadouros de rãs nas demais regiões do Brasil, motivo que tem prejudicado o crescimento dessa atividade no país (LIMA; CRUZ;
MOURA 1999; FERREIRA et al. 2002; CARRARO 2008; CRIBB, 2009; MOREIRA 2011).

A ranicultura no estado de Rondônia é desbravadora, e enfrenta problemas que afetam o desenvolvimento dessa atividade na região, principalmente quando se tratam de temas relacionados ao escoamento e comercialização da produção, ausência de frigoríficos e cooperativas especializadas no processamento e beneficiamento dos produtos e subprodutos da carne de rã, falta de assistência técnica especializada, carência de incentivos governamentais, alto preço dos insumos, poucas opções de acesso ao crédito, baixa procura dos produtos pelas comunidades locais, e falta de difusão de tecnologia para os ranicultores. Estes gargalos encontrados na cadeia produtiva de rã na região do estudo, do mesmo modo sucedem rotineiramente em diversas atividades do agronegócio no Brasil (LIMA; CRUZ; MOURA, 1999; WEICHERT; MELLO; ESPINDOLA, 2007; CRIBB et al., 2009; AFONSO, 2012). Ainda, Mathias e Scott (2004) relatam que a escassez do produto na entressafra e a falta de publicidade junto ao mercado consumidor dificultam o crescimento desse setor. Por outro lado, a carência de apoio de instituições credoras, o elevado custo com a produção; assistência técnica insuficiente; baixa qualificação dos produtores e dificuldade de acesso à tecnologia também, dificultam o desenvolvimento econômico da ranicultura (KUBITZA, CAMPOS, ONO et al., 2012; BORIN et al., 2013), contribuindo dessa forma com a desmotivação dos produtores que querem ingressar na atividade e pelos ranicultores remanescentes, e, por conseguinte, na insustentabilidade da produção de rã no estado de Rondônia e no país como um todo.

\section{CONCLUSÃO}

A criação de rãs no estado de Rondônia é caracterizada como atividade secundária para a 
geração de renda, atua no sistema Anfigranja, utiliza mão de obra familiar, e apresenta uma produção de pequena escala (5 ton.ano ${ }^{-1}$ ). Os produtores de rã alegam ser os principais gargalos para o setor, a falta de assistência técnica, altos custos com ração e a ausência de divulgação e incentivos para a atividade.

\section{AGRADECIMENTOS}

Agradecemos a Universidade Federal de Rondônia - UNIR pelo suporte logístico e instalações, aos proprietários dos ranários dos municípios de Rolim de Moura e de Monte Negro pelas informações fornecidas para o desenvolvimento desse trabalho.

Todos os autores declararam não haver qualquer potencial conflito de interesses referente a este artigo.

\section{REFERÊNCIAS}

AFONSO, A.M. Ranicultura se consolida com cadeia produtiva operando em rede interativa. Revista Visão Agrícola, v.11, p.33-35, 2012.

AGOSTINHO, C.; SILVA, M.; TORRES, R.; LIMA, S. Curvas de crescimento de rãs-pimenta, Leptodactylus labyrinthicus (SPIX, 1824). Revista da Sociedade Brasileira de Zootecnia, v.20, n.1, p.4754, 1991.

ANTONUCCI, A.M. Caracterização de agentes patogênicos virais e metazoários em rãs-touro, Lithobates catesbeianus, provenientes de ranários comerciais do Vale do Paraíba no estado de São Paulo, Brasil. São Paulo, SP. Dissertação de Mestrado. Instituto de Pesca do Estado de São Paulo; 2009.

BARDIN, L. Análise de conteúdo. Lisboa: Edições 70, p 229, 1977.

BORIN, E.C.P.; LIMA, F.G.F. Uma abordagem do associativismo: o estudo de caso da ranicultura em Guaratiba/RJ. Revista da Polêm!ca, v.12, n.4, p.740749, 2013.

BRAGA, L.G.T.; LIMA, S.L. Influência da temperatura ambiente no desempenho da rã-touro, Rana catesbeiana (Shaw, 1802) na fase de recria. Revista Brasileira de Zootecnia, v.30, n.6, p.16591663, 2001.
CARRARO, K.C. Ranicultura: um bom negócio que contribui para a saúde. Revista da FAE, v.11, n.1, 2016.

CARVALHO, L.T. Diagnóstico da competitividade na cadeia produtiva de carne de rã-touro no Estado do Rio de Janeiro. Viçosa, MG. Tese de Pós-Graduação. Universidade Federal de Viçosa-UFV;2011.

CASTRO, C.S de. Taxas e frequências de alimentação na produção de rã-touro em baias inundadas. Botucatu, SP. Dissertação de Mestrado. Universidade Estadual Paulista-UNESP; 2010.

COLOMBANO, N.C. Suplementação alimentar com vitamina $\mathrm{C}$ e desempenho zootécnico de girinos de rãtouro (Rana catesbeiana). Jaboticabal, SP. Dissertação de Mestrado. Universidade Estadual Paulista-UNESP; 2005.

CORRÊA, C.F.; SCORVO-FILHO, J.; D TACHIBANA L.; GERVÁZIO LEONARDO, A.F. Caracterização e situação atual da cadeia de produção da piscicultura no Vale do Ribeira. Informações Econômicas, v.38, n.5, p.30-36, 2008.

CRIBB, A.Y. Avaliação e transferência de tecnologia: os contornos de um projeto de dinamização da inovação na cadeia da rã. In: Congresso da Sociedade Brasileira de Economia, Administração e Sociologia Rural, 47, Brasília, DF, Anais. SOBER, p.1-221, $2009 . \quad$ Disponível em: http://www.alice.cnptia.embrapa.br/alice/handle/doc/ 662295. Acesso em: 15/06/2017.

CRIBB, A.Y.; AFONSO, A.M.; MOSTÉRIO, C.R.F. Manual técnico de ranicultura. 1 ed., Brasília, DF: Embrapa, p.73, 2013.

CRIBB, A.Y.; DE CARVALHO, L.T.; MENDONÇA, R. C. S. O consumo de carne de rã: caracterização, tendências e perspectivas. 21 ed., Rio de Janeiro: Embrapa - Agroindústria de Alimentos, p.18, 2009.

DE OLIVEIRA, E.G. Ranicultura: novos desafios e perspectivas do mercado. Ciência Animal, v.25, n.1, p.173-186, 2015.

DIAS, D.D.C.; DE STÉFANI, M.V.; FERREIRA, C.M.; FRANÇA, F.M.; RANZANI- PAIVA, M. J. T.; SANTOS, A.A. Haematologic and immunologic parameters of bullfrogs, Lithobates catesbeianus, fed probiotics. Aquaculture Research, v.41, n.7, p.10641071, 2010.

FAO, Food and Agriculture Organization of the United Nations. Fishery and Aquaculture Statistics. 2008/FAO annuaire. Rome: FAO, 72p., 2010. Disponível em: 
http://www.fao.org/fishery/statistics/es. Acesso em: 15/04/2017.

FEIX， R.D.; ABDALLAH， P.R.; FIGUEIREDO, M.R.C. Resultado econômico da criação de rã em regiões de clima temperado, Brasil. Informações Econômicas, SP, v.36, n.3, p.70-82, 2006.

FERREIRA, C.M.; PIMENTA, A.G.C.; PAIVANETO, J.S. Introdução à ranicultura. Boletim Técnico do Instituto de Pesca, v.33, p.1-15, 2002.

FIGUEIREDO, M. R. C.; LIMA, S. L.; AGOSTINHO, C.A.; BAÊTA, F. da C.; WEIGERT, S.C. Estufas climatizadas para experimentos ambientais com rãs, em gaiolas. Revista Brasileira de Zootecnia, v.30, n.4, p.1135-1142, 2001.

FONTANELLO, D.; PENTEADO, L.A.; WIRZ, R.R.; SOARES, H.A. Criação experimental de rã-touro (Rana catesbeiana Shaw, 1802) em gaiolas. VI ENAR, Encontro Nacional de Ranicultura, Rio de Janeiro, Brasil, p.201-228, 1988.

FONTANELLO, D.; WIRZ, R.R.; SOARES, H.A., FREITAS, E.; CAMPOS, B.D.; FERREIRA, C.M. Comparação de quatro sistemas de engorda de rãstouro (Rana catesbeiana Shaw, 1802): tanque-ilha, confinamento, anfigranja e gaiolas. Boletim do Instituto de Pesca, v.20, p.43-58, 1993.

GOMES, C. de O.E. Perspectivas para a ranicultura no Estado de Rondônia. Cacoal, RO. Bacharelado em Administração. Universidade Federal de Rondônia, 2008.

Instituto Brasileiro de Geografia e Estatística. 2015. Pesquisa da Pecuária Municipal - PPM: IBGE. Disponível em https://biblioteca.ibge.gov.br/visualizacao/periodicos/ 84/ppm_2015_v43_br.pdf. Acesso em 20/06/2017.

Instituto Brasileiro de Geografia e Estatística. 2017. Estados@: Rondônia [Internet]: IBGE. Disponível em:

http://www.ibge.gov.br/estadosat/perfil.php?sigla=ro. Acesso em 20/06/2017.

Instituto Brasileiro do Meio Ambiente e dos Recursos Naturais Renováveis. 2009. Estatística da pesca 2007. Brasil: Grandes regiões e unidades da federação. Brasília: IBAMA, p.113.

Instituto Nacional de Meteorologia - INMET [2017]. Disponível:

http://www.inmet.gov.br/portal/index.php?r=agromet eorologia/boletimAgroclimatologico. Acesso em $06 / 11 / 2017$
JUNIOR, J.F.; DE STÉFANI, M.V. Desempenho e parâmetros metabólicos de rã-touro, Rana catesbeiana, alimentada com diferentes rações comerciais. Acta Scientiarum. Animal Sciences. v.27, n.3, p.377-382, 2005.

KAUFMANN, J.C. A entrevista compreensiva: um guia para pesquisa de campo. Petrópolis, Maceió, AL: Vozes, p.202, 2013.

KUBITZA, F.; CAMPOS, J.L.; ONO, E.A.; ISTCHUK, P.I. Panorama da piscicultura no Brasil: Estatísticas, espécies, pólos de produção e fatores limitantes à expansão da atividade. Panorama da aquicultura, v.22, n.132, p.14-25, 2012.

LIMA, S. L.; AGOSTINHO, C. A. A criação de rãs. Rio de Janeiro, RJ: Publicações Globo Rural, p.187, 1989.

LIMA, S.L.; AGOSTINHO, C.A. A tecnologia de criação de rãs. Viçosa, MG: Universidade Federal de Viçosa. Imprensa Universitária, p.166, 1992.

LIMA， S.L.; CRUZ, T.A.; MOURA, O.M. Ranicultura: análise da cadeia produtiva. Viçosa, MG. Editora Folha de Viçosa, p.172, 1999.

LIMA, S.L; CASALI, A.P; AGOSTINHO, C.A. Desempenho zootécnico e percentual de consumo de alimento de rã-touro (Rana catesbeiana) na fase de recria (pós-metamorfose) do sistema anfigranja. Revista Brasileira de Zootecnia, v.32, n.3, p.505$511,2003$.

MANSANO, C.F.M.; STÉFANI, M.V. D.; PEREIRA, M.M.; MACENTE, B.I. Non-linear growth models for bullfrog tadpoles. Ciência e Agrotecnologia, v.36, n.4, p.454-462, 2012.

MAZZONI, R. (Ed.) Ranicultura: manual básico para inversores. Montevidéo: Proyecto Ranicultura Dinara -IIP, 2001. Disponível em http://www.mgap.gub.uy/sites/default/files/multimedi a/1953_ranicultura_manual.pdf. Acesso em 20/09/2017

MOREIRA, C.R. Análise Econômica da Ranicultura: Viabilidade Individual e Integrada de Operações. São Paulo, SP. Dissertação de Mestrado. Instituto de Pesca - IP; 2011.

MORETTO, G.; RODRIGUES, C.; CRIBB, A.; FURTADO, A. L. dos S. Áreas potenciais para a criação de rã-touro-gigante (Lithobates catesbeianus Shaw, 1802) na região Sul do Brasil. In: Congresso Interinstitucional de Iniciação Científica, 7, Anais, Campinas, SP: IAC, 2013. p.1-8. Disponível em: 
http://www.alice.cnptia.embrapa.br/alice/handle/doc/ 970935. Acesso em 11/07/2017.

OLIVEIRA, G.A. Instalação de ranário. In: III Encontro Nacional de Ranicultores, 3, Uberlândia, MG. Universidade Federal de Uberlândia, p.41-57, 1982.

OLIVEIRA, M.M. Como fazer pesquisa qualitativa. 3 ed., Petrópolis, RJ: Vozes, p.181, 2008.

OSTRENSKY, A.; BORGHETTI, J.R.; SOTO, D. Aqüicultura no Brasil: o desafio é crescer. Brasília: FAO, 2008. Disponível em: http://projetopacu.com.br/public/paginas/202-livroaquicultura-no-brasil-o-desafio-e-crescer.pdf. Acesso em 20/06/2017.

RANÁRIO AURORA. Cultura da rã-gigante touro, Rana catesbeiana. Rio de Janeiro, RJ: Editora Ranário Aurora, p.58, 1938.

REIS, E.A.; REIS, I. A. 2002. Análise Descritiva de Dados. Relatório Técnico do Departamento de
Estatística da UFMG, p. 36. Disponível em: http://www.est.ufmg.br/portal/arquivos/rts/rte0202.pd f. Acesso em 03/03/2017.

RODRIGUES, C.; QUARTAROLI, C.; CRIBB, A.; BELUZZO, A. 2010. Áreas potenciais para a criação de rã-touro gigante Lithobates catesbeianus (Shaw, 1802) na região Sudeste do Brasil. Embrapa Monitoramento por Satélite - Boletim de Pesquisa e Desenvolvimento. Campinas, SP, p. 38. Disponível em:

https://www.infoteca.cnptia.embrapa.br/bitstream/doc /884549/1/BPD12.pdf. Acesso em 11/07/2017.

VIZOTTO, L.D. Aspectos técnicos da ranicultura. In: ENCONTRO NACIONAL DE RANCILTURA, 1, 1978, Anais. Brasília: MA/SUDEPE/CNA/IBDF/SAP, p. 27-60, 1979.

WEICHERT, M.A.; MELLO, S.R.P.; ESPINDOLA, L.M.O. Consumo de tilápias e rãs nas cidades do Rio de Janeiro e Niterói. Panorama da Aquicultura, Rio de Janeiro, v.17, n.102, p.37-41, 2007. 